\title{
Gefährliche Gruppen. Zur Personifizierung sozialer Probleme
}

\author{
Dörte Negnal
}

Eingegangen: 30. Juni 2020 / Angenommen: 14. September 2020 / Online publiziert: 28. Oktober 2020 (C) Der/die Autor(en) 2020

Zusammenfassung Gelten soziale Gruppen als Bedrohung für eine allgemeine Bevölkerung, kreist die Problematisierung weniger um die aktuelle Situation oder gesellschaftliche Bedingungen, sondern soziale Probleme werden personifiziert. Im Beitrag wird die Herstellung solcher Problemgruppen als kategoriale Verdichtung diskutiert. Unterstrichen wird dabei, dass die Beteiligungen der Akteur*innen über einen reaktionären Opferstatus hinausreichen und sich kollektive Akteur*innen über die kategoriale Verdichtung formieren. Plädiert wird dafür, Problematisierungen in Konstellationen aus Allgemeinheit, problematisiertem Kollektiv und Problemgruppe zu fassen. Dafür wird eine akteurs- und prozessorientierte Perspektive bemüht, denn dem Phänomen der Personifizierung sozialer Probleme auf die Spur zu kommen, bedeutet, ihr Werden, die Prozeduren und die Beteiligten - die Problematisierten wie die Problematisierenden - mit ihren Beiträgen dahingehend zu untersuchen, wie die bestimmende Kategorisierungsarbeit jeweils geleistet wird. Diese Perspektive wird mit Bezug auf interaktionistische Studien und Ansätze aus den Science and Technology Studies theoretisch hergeleitet und mit Fällen aus eigenen Forschungen empirisch begründet.

Bei dem vorliegenden Beitrag handelt es sich um eine überarbeitete Version meiner am 04.12.2019 gehaltenen Antrittsvorlesung an der Universität Siegen.

D. Negnal $(\bowtie)$

Universität Siegen, Adolf-Reichwein-Straße 2, 57068 Siegen, Deutschland

E-Mail: doerte.negnal@uni-siegen.de 


\section{Dangerous Groups. About the Personification of Social Problems}

Abstract If social groups are seen as a threat to a general population, social problems are pinned on people rather than seen as a situational or structural challenge. The article discusses the construction of such problem groups as collective membership categorization. It is emphasized that the participation of the actors goes beyond a reactionary victim status. Collective actors are formed through categorical compression. Therefore, problematizations ought to be analyzed in constellations of general public, problematized collective and problem group. For this purpose, an actor- and process-oriented perspective seems to be useful. This perspective is derived theoretically with reference to interactionist studies and approaches from Science and Technology Studies. To study the phenomenon of personification of social problems means to examine its becoming, the procedures and participants- the problematized as well as the problematizing-with the members' accountings for being dangerous. This is shown empirically with cases from my own research on problem groups.

\section{Einleitung: Soziale Probleme und soziale Gruppen}

Soziale Probleme - etwa Armut, Drogensucht oder Kriminalität - sind soziale Phänomene, die öffentlich thematisiert und institutionell bearbeitet werden. Unter der Prämisse, sie als Ausdruck gesellschaftlicher Verhältnisse zu verstehen (Bellebaum und Braun 1974), werden soziale Probleme zumeist als soziale Ungleichheitslagen analysiert. Gesellschaftliche Verhältnisse bestehen jedoch nicht einfach. Sie werden geschaffen. Insofern hat sich die Perspektive durchgesetzt, soziale Probleme als soziale Produktion zu begreifen. In dieser konstruktivistischen Hinsicht sind drei Ansätze formuliert worden: das Kokonmodell von Schetsche, der Ansatz des Doing Social Problems, den Groenemeyer aufgearbeitet hat, und der Blick auf die Rolle staatlicher Instanzen, deren Verwicklungen vor allem von der Kritischen Kriminologie aufgegriffen worden sind.

Damit sich soziale Probleme entwickeln können, braucht es nach Schetsche (2014, S. 85-100) kollektive Akteur*innen, die ein „Problemmuster“ (ebd., S. 52) formulieren. Schetsche konstatiert, dass wenig dazu bekannt ist, wie sich diese Gruppen an der Thematisierung eines Problems beteiligen, nur, dass sie entscheidenden Einfluss auf die Durchsetzung einer Problemwahrnehmung haben (ebd., S. $85 \mathrm{ff}$.). Er geht von kollektiven Akteur*innen als eine oder mehrere soziale Gruppen aus, die sich mit Motiven, Zielen und Handlungsstrategien der Problematisierung widmen (Schetsche 1996, S. 39 ff.). Dabei bleiben häufig diejenigen, die das soziale Problem betrifft, außen vor (Schetsche 2014, S. 87 f.). Problemgruppenzugehörige beteiligen sich jedoch an ihrer Konstruktion. Sie sind nicht allein ,fremddeklarierte Opfer“ (ebd., S. 87) einseitiger Zuschreibungen. Sie ko-produzieren die Zuschreibungen. Es kann also nicht von einer kollektiven Intention ausgegangen werden, denn kollektive Akteur*innen formieren sich erst in interaktiven Prozessen. 
Groenemeyer fasst die Konstruktion sozialer Probleme als soziale Produktion ${ }^{1}$ auf, die sich vor dem Hintergrund gesellschaftlicher Bedingungen ereignet (2001a, S. 17). Soziale Probleme entstehen dann in institutionellen Kontexten staatlicher und nicht-staatlicher Initiativen und werden dort reproduziert. Der von ihm 2010 herausgegebene Band zu sozialen Problemen schließt an Arbeiten der US-amerikanischen Soziolog*innen zum Doing Social Problems an (Kitsuse und Spector 1973; Gusfield 1989; Miller und Holstein 1993), in denen herausgearbeitet wird, wie sich die Bearbeiter*innen ein soziales Problem in ihrer Tätigkeit aneignen und es als relevantes Tätigkeitsfeld reproduzieren. Im Doing verstetigt sich die öffentliche Thematisierung, die sich dann in Formen sozialer Kontrolle niederschlägt (Groenemeyer 2010).

Dies ist auch dem Umstand zugetan, dass es sich bei unerwünschten Zuständen - als Ausdruck sozialer Probleme - um soziale Situationen handelt, die nicht angestrebt, vielmehr verhindert oder gemindert werden sollen. Die dabei als Klientel Identifizierten bleiben Subjekte der Bearbeitung. Die gewichtige Rolle staatlicher Instanzen haben Vertreter*innen der Kritischen Kriminologie herausgestellt, so etwa, wie abweichendes Verhalten aus Zuschreibungsprozessen staatlicher Institutionen resultiert und sich in ,abweichenden Karrieren“ (etwa Quensel 1970; Scheerer 1978) und Mechanismen sozialer Kontrolle verfestigt. Daher sind die Agent*innen sozialer Kontrolle zu untersuchen (Scheerer 2000; Wehrheim 2000; Peters 2000; Hess 2015). So trägt die wissenschaftliche Beschäftigung hierzu bei. Häufig bleiben aber auch hier Vertreter*innen der Identifikation der Zugehörigen und ihrer institutionellen Bearbeitung verhaftet (Hess 1993; Dollinger 2010). Insofern zeigt sich in den Beiträgen, dass soziale Probleme Diskursfelder bilden.

Analytisch liegt es dann nahe, zu rekonstruieren, wann welche Phänomene wie als problematisch erscheinen, denn der Gegenstand - das Soziale am Problem - fordert ein, die Interaktionen im gesellschaftlichen Gefüge nachzuvollziehen. Um derlei Wechselwirkungen zu untersuchen, skizzieren die oben genannten Ansätze kollektive Akteur*innen, die sich daran abarbeiten, ein Phänomen öffentlich zu setzen und institutionell zu bearbeiten. Schetsche zeichnet ein Verlaufsmodell, dessen Schwerpunkt bei der medialen Aufbereitung liegt (1996, 2014). Groenemeyer vollzieht die Genese sozialer Probleme in gesellschaftlich-historischen Kontexten nach (2001b, S. 150), um soziale Probleme an gesellschaftliche Bedingungen rückzubinden (u.a. 2001a, b; Groenemeyer und Wieseler 2008). Richtig ist, dass der Kontext und damit zeitliche Bezüge Berücksichtigung erfahren müssen, aber nicht losgelöst vom Produktionsprozess des sozialen Problems (Scheffer 2008). Peters nimmt die Instanzen sozialer Kontrolle in den Blick (2000, sowie in diesem Heft). Allerdings betrachten die genannten Autor*innen dabei nicht, wie sich die kollektiven Akteur*innen im Problematisierungsgeschehen herausbilden. Die am Phänomen Beteiligten tauchen entweder als vom Problem Betroffene auf, die das soziale Problem repräsentieren Arme, Drogensüchtige, Kriminelle - oder als meist professionelle Bearbeiter*innen des Problems - Sozialarbeitende, Psycholog*innen, Polizist*innen. Die als ,folks devils“ (Cohen 1972) oder ,troubled persons“ (Gusfield 1989) Benannten sind in ih-

\footnotetext{
1 Wie kaum ein zweiter zeichnet Groenemeyer die Strömungen in der Soziologie sozialer Probleme nach. Eine sehr gelungene Darstellung findet sich in der 2. Auflage des Handbuchs (2012, S. 17-116).
} 
rer öffentlichen Thematisierung wie im institutionellen Werden betrachtet worden, nicht jedoch, wie sie als soziale Gruppen selbst entstehen. Das liegt auch daran, dass ihnen keine eigene Agentenschaft zugesprochen wird. Vielmehr bleiben sie im Doing, dem Handlungsvollzug sozialer Probleme, die Betroffenen, um die herum sich die Professionellen gruppieren (Dollinger 2010). Und in dieser Reproduktion der Identifizierung, Thematisierung, Kategorisierung und Bearbeitung der Zugehörigen - erscheinen uns die Setzungen fast natürlich bzw. selbstverständlich.

Doch Problemzuschreibungen sind weder im Individuum angelegt noch allein Sache von staatlichen Einrichtungen oder diskursiven Strategien. Die gesellschaftlichen Verhältnisse in den Blick zu bekommen heißt, die Konstellationen zu untersuchen, in denen sie sich ereignen. Problematisierungen formieren sich im gesellschaftlichen Miteinander. Dabei geht es weniger um antagonistische Gruppenzuschnitte von Problematisierenden und Problematisierten. Akteur*innen, die das Soziale am Problem kreieren, tun dies in sozialen Situationen und Prozessen. Sie klagen an, wehren sich gegen Zuschreibungen, fordern auf, tätig zu werden usw. Die das soziale Problem Betreffenden sind nicht allein Klient*innen, die in einem reaktionären Opferstatus adressiert werden - die Armen, die Drogensüchtigen. Ebenso kann ihnen aktive Tätigkeit zugeschrieben werden, z. B. in Form von politischem Aktivismus oder Gewalthandeln. Insofern erscheint es vielversprechend, Problematisierungen in Konstellationen aus Allgemeinheit, problematisiertem Kollektiv und Problemgruppe zu fassen. ${ }^{2}$ Hier zeigt sich bislang eine Leerstelle in der Analyse sozialer Probleme.

Ultras, Hooligans, Salafisten, der Schwarze Block oder die Identitären sind Beispiele für soziale Gruppen, die in der öffentlich-gesellschaftlichen Wahrnehmung als gefährliche Zusammenschlüsse erscheinen. Ihre Zugehörigen sollen erkannt, beobachtet und ihre Taten verhindert bzw. strafrechtlich verfolgt werden, denn sind es nicht die Mitglieder einer solchen problematisierten Gruppe, die bedrohliche Situationen schaffen? Aufmärsche von Neonazis oder Identitären in den letzten Jahren, Hooligans in der Kölner Innenstadt 2014 oder die Anschlagserie 2015 in Paris liefern eindrückliche Beispiele für Populismus, Gewalt, Kriminalität und Terror. Dabei wird weniger nach der Situation selbst, sondern mehr danach geschaut, wer ihre Urheber*innen sind. Denn es handelt sich um unerwünschte Zustände, die potenziell jede* $n$ angehen können, weil sie vermeintlich die Sicherheit aller Gesellschaftsmitglieder bedrohen. Immer wenn eine Allgemeinheit sich in Betroffenheit wähnt, wird weniger das soziale Problem benannt. Das Problem wird gerade nicht als das ,eigene", in der gesellschaftlichen Allgemeinheit zu lokalisierende Problem entworfen, weil sich eine allgemeine Bevölkerung abgrenzt, wenn sie andere problematisiert. So rückt eine soziale Gruppe in den Fokus, von der das Problem auszugehen scheint. Am deutlichsten zeigt sich dieser Mechanismus bei Phänomenen der Sicherheit und Ordnung. In diesen Fällen werden aus Betroffenen Tat-Kräftige, die scheinbar das Problem ausmachen und die in der Bedrohung, die von ihnen auszugehen scheint, wirkmächtig sind.

Dies wird durch einen weiteren Umstand begünstigt. Es lassen sich vermeintlich eindeutige Täterschaften identifizieren: die neuen Rechten, die libanesischen Clans,

\footnotetext{
${ }^{2}$ Ich danke den Mitgliedern des Arbeitskreises politische Ethnographie für den langjährigen wertvollen Austausch nicht nur zu diesem Gedanken.
} 
die Salafisten oder Syrien-Rückkehrer. Zumeist werden folgende Fragen adressiert: Welche Motive haben sie? Wie sind die Gruppen strukturiert? Welche Lösungsmöglichkeiten bieten die Bearbeitungsinstanzen, etwa Polizei, Soziale Arbeit und Politik an? Solche Fragen erschaffen soziale Gruppen, weil sie nach Merkmalen fragen. Daraus ergeben sich zwei spannende Effekte: Zum einen können sich Individualisierungen Bahn brechen. Beispielgebend ist hier die Frage danach, wie sich einzelne Täter*innen radikalisiert haben. In Deutschland wurde z. B. versucht den Weg von Anis Amri, jenem Fahrer, der einen zuvor gestohlenen LKW in den Weihnachtsmarkt am Berliner Breitscheidplatz steuerte, nachzuzeichnen. Zum anderen fließen Kulturalisierungen in die Bestimmungen der Problematisierten mit ein, vorrangig ethnische, klassen- und milieuspezifische Aspekte. Was beim Problematisieren unerwünschter Zustände also passiert, ist die Personifizierung sozialer Probleme. Es werden soziale Gruppen kreiert, für die sich dann Repräsentant*innen bestimmen lassen. Das schließt an Groenemeyer dahingehend an, dass Repräsentationen Grundlage für die Institutionalisierung neuer Realitäten sind (Groenemeyer 2001b), mit dem Effekt, dass sich soziale Kontrollmechanismen ausweiten.

Problematisierungen stoßen Wechselwirkungen an. Sie sind sozial organisierte und kommunikative Prozeduren (Peters 2000), in deren Zuge soziale Gruppen als problematisch entworfen werden und sich als Problemgruppen etablieren (Negnal 2019b, S. 18).

Die Betrachtung des Sozialen schlüsselt Groenemeyer (2001a) in dreierlei Hinsicht auf: interaktionistisch, worin er die Mikropolitiken verortet, gesellschaftlich, insofern er die gesellschaftlichen Bedingungen bemüht, und zu letzterem in Korrespondenz stehend staatlich, womit er vor allem soziale Kontrolle durch staatliche Instanzen aus Politik und Verwaltung benennt, die soziale Probleme instrumentalisieren und organisieren (2001a, S. 14). Diese drei Dimensionen verschränken sich in der Analyse der Problematisierung sozialer Gruppen. Das Agieren der Beteiligten wird interaktionistisch rekonstruiert, ihre gesellschaftliche Einbettung als Allgemeinheit, problematisiertes Kollektiv oder Problemgruppe betrachtet und staatliche Verwicklungen in Verfahren und Prozessen werden berücksichtigt.

Vor diesem Hintergrund nimmt der vorliegende Beitrag begriffliche Einfassungen vor, um die Leerstelle der Analyse sozialer Probleme aufzuarbeiten. Unterstrichen wird dabei, dass die Beteiligungen der Akteur*innen über einen reaktionären Opferstatus hinausreichen und sich kollektive Akteur*innen über eine kategoriale Verdichtung formieren. Dem Phänomen der Personifizierung sozialer Probleme auf die Spur zu kommen, bedeutet, ihr Werden, die Prozeduren und die Beteiligten die Problematisierten wie die Problematisierenden - mit ihren Beiträgen dahingehend zu untersuchen, wie die bestimmende Kategorisierungsarbeit jeweils geleistet wird. Den Ausgangspunkt findet eine solche Analyse in der sozialen Situation, bei ihren Akteur*innen und deren Beiträgen, die in den Prozess der Setzung eingehen. Insofern werden für die Herleitung des Ansatzes relevante Aspekte aus interaktionistischen Zugängen gefiltert (Abschn. 2) und die Gruppenzuschnitte des problematisierten Kollektivs und der Problemgruppe charakterisiert (Abschn. 3). Anschließend lege ich die für den Produktionsprozess der Problemgruppe zentralen Prozesse dar (Abschn. 4) und schließe mit einer Schärfung der Perspektive auf die Konstella- 
tion aus allgemeiner Bevölkerung, problematisiertem Kollektiv und Problemgruppe (Abschn. 5).

\section{Interaktionistische Ansätze: Problematisierung als kategoriale Verdichtung}

In interaktionistischen Ansätzen ist die Personifizierung sozialer Probleme bislang vor allem im Engagement und Umgang mit abweichendem Verhalten gefasst worden. ${ }^{3}$ Hier sind drei Aspekte für die Herstellung von problematisierten sozialen Gruppen relevant: (1) Der Problematisierungsprozess erfolgt öffentlich. Ein Publikum bezeugt die Problemzuschreibungen und die Problematisierten fügen sich in diesen Prozess ein. (2) Eine soziale Gruppe wird darin zum Objekt, wie sie in Moraldebatten verhandelt wird. (3) Die Relevanz des sozialen Problems wird dann durch die problembearbeitenden Instanzen hergestellt.

1. Die prominentesten Arbeiten zur Einfügung einer sozialen Gruppe in einen öffentlichen Problematisierungsprozess stammen von Howard S. Becker (2014 [1963]) und Erving Goffman (1990). Während Becker mit dem Konzept des Moralunternehmertums stark macht, wie es Akteur*innen gelingt, Regeln zu setzen, und sich diejenigen, die von den Regeln abweichen, in ihr Außenseiter-Dasein einfügen, zeigt Goffman, wie potenziell jede*r zur Außenseiter*in diskreditiert werden kann, weshalb sich Akteur*innen ein breites Repertoire an Strategien aneignen, um diese Gefahr einzuhegen. Beide Autoren gehen davon aus, dass das abweichende Verhalten ein Stigma sei, das die Betreffenden als anerkannte Gesellschaftsmitglieder disqualifiziert und dass sie deshalb versuchen, diesen Makel zu kaschieren und mit dem Ausgegrenztwerden umzugehen. Die Diskreditierten fügen sich dann in den Prozess der Problematisierung als Betroffene ein. Hierfür bedarf es einer Öffentlichkeit, in der bestehende Normen als unzureichend bzw. ausbaufähig qualifiziert werden und diejenigen, die durch ihr Verhalten den vermeintlichen Stein des Anstoßes bilden, als Außenseiter*in markiert werden können (Becker 2014 [1963]).

2. Das Herstellen einer Öffentlichkeit vollzieht sich dabei zum einen über Instanzen sozialer Kontrolle, die angerufen werden, tätig zu werden. Besorgte Bürger*innen und professionelle Problembearbeiter*innen (Polizist*innen, Sozialarbeitende, Lehrer*innen) thematisieren das abweichende Verhalten, indem sie das Auftreten seiner Urheber*innen problematisieren. Cohen hat in den 1950er-Jahren am Beispiel der Mods und Rocker (Jugendliche, die mit Mopeds gefahren sind) aufgezeigt, wie die als abweichend Deklarierten zum Objekt medialer Debatten werden, in denen Fragen zum moralisch ,richtigen“ Verhalten ausgetragen werden (Cohen 1972). Das Abarbeiten am vermeintlich abweichenden Gegenüber benötigt die mediale Berichterstattung zur Dramatisierung, wie wir es beispielsweise beim Thema Jugendkriminalität kennen. Indem Ereignisse zu skandalösen Vorfällen werden, können die als abweichend Wahrgenommenen kategorial verdichtet

\footnotetext{
3 Ausführlich hierzu Negnal (2019b).
} 
werden und sie erscheinen als eine soziale Gruppe: z.B. als jugendliche Gewalttäter*innen. Bei einem symbolischen Kreuzzug (Gusfield 1986) werden nicht die Sache, sondern die Parteien zentral.

3. Mit der Problematisierung werden schließlich Bearbeitungskapazitäten für das soziale Problem bereitgestellt. Die Problembearbeiter*innen aktualisieren das Problem in der Bearbeitung gemeinsam mit ihren Klient*innen (Gusfield 1989; Miller und Holstein 1989) und reproduzieren es so. Das ist im Ansatz des Doing Social Problems als Selbstreferenzialität herausgearbeitet worden (zusammenfassend Groenemeyer 2010).

Es gibt also bereits seit den 1950er-Jahren Studien zur Problematisierung, in denen soziale Gruppen thematisiert werden. Doch sind diese als Auslösende, Betroffene und Klient*innen, nicht jedoch selbst als Phänomen konzeptioniert worden. Dies hängt mit den Abgrenzungsbemühungen gegenüber strukturfunktionalistischen Ansätzen und dem Bedeutungsgewinn interaktionistischer Ansätze zusammen. Letztere haben den Blick auf Prozessgeschehen freigegeben, indem sie Zuschreibungspraktiken untersuchten. Dies wurde unter anderen von Becker (2014) und Goffman (1973a) in den Karrierebegriff gegossen. Dennoch ist den Ansätzen eine auf das Individuum fokussierte Sicht gemein. Der*die Einzelne ist von Moralunternehmertum bedroht und versucht mit Techniken der Informationskontrolle das Stigma abzuwehren. Indem das abweichende Verhalten dramatisiert und seine Urheber*innen öffentlich thematisiert werden, erhalten diese den Charakter eines Objekts, über das sich dann diskutieren lässt und an dem sich abgearbeitet wird - diskursiv wie institutionell. Damit festigt sich die problematisierte Kategorie hin zur sozialen Gruppe von Abweichenden, für die konkrete Bezeichnungen gefunden werden.

Um der Kategorienverdichtung von der sozialen Gruppe zum sozialen Problem näherzukommen, können zwei wichtige Lehren aus den interaktionstheoretischen Ansätzen gezogen werden: Erstens haben sie den Blick auf Prozessgeschehen freigegeben, ohne dieses selbst zum Gegenstand der Analyse zu machen. Zweitens haben sie kollektives Verhalten angesprochen (etwa Gusfield 1986 zur Temperance Bewegung), ohne die Kollektive selbst in den analytischen Fokus zu rücken.

Dennoch sind relevante Aspekte angedeutet: das reaktive Einfügen der Betreffenden und damit ihr Engagement im Problematisierungsprozess, die Öffentlichkeit, die Objektivierung, um soziale Gruppen zum Gegenstand der Debatte zu machen und die Relevanz setzende Reproduktion sozialer Probleme im Zuge ihrer Bearbeitung. Ich möchte im Folgenden zwei Aspekte mobilisieren - zum einen das Engagement der Betreffenden und zum zweiten ihre Objektivierung. Um der Kategorienverdichtung von der sozialen Gruppe als soziales Problem näherzukommen, ist eine begriffliche Einfassung mit zwei Anleihen notwendig: die kategoriale Zugehörigkeit aus der Humandifferenzierung und der Objektcharakter aus den Science and Technology Studies.

\subsection{Soziale Gruppe als kategoriale Zugehörigkeit}

Mit Bezug auf Hirschauer (2017) spreche ich von einer sozialen Gruppe im Sinne einer sozialen Mitgliedschaft, die entsteht, wenn eine Kategorie (z.B. Ethnizität, 
religiöse Strömung oder auch Devianz) auf eine Person angewandt wird (Negnal 2019b, S. 27). Beispielsweise drückt die Bezeichnung „Salafisten“ eine kategoriale Zugehörigkeit aus. Die dieser Kategorie Zugehörigen werden zu einer vermeintlichen Handlungsgruppe, indem eine kategorisierende Bezeichnung im Umlauf ist, die eine soziale Einheit annimmt oder vielmehr setzt. „Die Salafisten“ sind jedoch keine Gruppierung mit einem Wir-Gefühl oder einer Agenda. Solche, auf Gruppierungen bezogenen Zugehörigkeiten, etwa Fangruppierungen beim Fußball, bestimmt Hirschauer (2017, S. 30) als relational.

Die soziale Gruppe, die im Zuge von Problematisierungen entsteht, bezeichnet also kategoriale Zugehörigkeiten statt Personenkreise. An diese Kategorie können sich Stigmatisierungen anheften. Die bezeichnete Person wird zur Repräsentant*in einer vermeintlichen Gruppierung und ihre verschiedenen Facetten werden auf einen Master-Status hin zugespitzt, zumeist auf den der Devianz. Dies gelingt nun eben nicht entlang von Gruppendynamiken, sondern durch die Objektivierung der sozialen Gruppe. Die soziale Gruppe wird selbst zum Phänomen.

\subsection{Objektcharakter}

Die Science and Technology Studies haben einen für die Analyse sozialer Probleme gewinnbringenden Aspekt herausgestellt. Rheinberger (1997) etwa ging der Frage nach, wie Forschungsgegenstände in den Naturwissenschaften entstehen. Ein Forschungsbereich, in seinem Fall die Genforschung, kreiere fortwährend genügend offene Fragen, denen sich weitere Forschungsteams widmen können, weil das Objekt der Forschung, das Gen, in seiner Bestimmung notwendigerweise unscharf bleibt. Es wird zu einem ,epistemischen Ding“, an das sich Wissenspraktiken anheften (Rheinberger 2006). Epistemische Dinge erzeugen eine Produktivität: Neugier und Spannung sorgen dafür, dass sich Forschende immer weiter in dem Feld engagieren. Es entstehen neue Disziplinen und Arbeitsbereiche, Methoden und Differenzierungen.

Auf unseren Fall bezogen können nun problematisierte soziale Gruppen zu epistemischen Objekten avancieren, die neue Maßnahmen, Techniken und Arbeitsbereiche erfordern, beispielsweise Mapping-Projekte beim Bundeskriminalamt oder die Einrichtung eines gemeinsamen Terrorabwehrzentrums (GTAZ).

Eine problematisierte soziale Gruppe kann einen Objektcharakter erhalten, weil das Potenzial der Problematisierten betont wird, sich als Phänomen auszuweiten: Gruppen könnten sich zusammenschließen, Ausschreitungen könnten die Bedrohungslage verschärfen usw. Beispiel gebend sind die Ausschreitungen von Hooligans und Neonazis unter der Bezeichnung HOGESA, die in der Kölner Innenstadt gegen Salafisten mobil machten. Multiple Möglichkeiten lassen sich nur bedingt berechnen, aber sie ermöglichen die weitere Bearbeitung: dem Nicht-Wissen zu Hooligans und Ultras in Fußballstadien, Neonazis und Neuen Rechten wird in zahlreichen

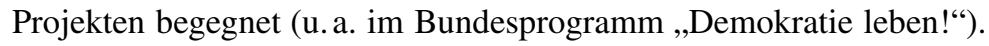

Aus den beiden konzeptuellen Einfassungen der sozialen Gruppe - als kategoriale Zugehörigkeit mit einem Objektcharakter, der potenziell viele Möglichkeiten bietet - lässt sich ein analytischer Zugriff für die Problematisierung sozialer Gruppen formulieren, wenn die kategoriale Zugehörigkeit nicht nur in Fremdzuschreibungen 
konzeptionalisiert wird. Mit einer aktiven Wendung der Mitgliedschaftskategorie engagieren sich die Mitglieder (Sacks 1972, 1992) und lassen sich so im Problematisierungsprozess unterscheiden.

\section{Problematisiertes Kollektiv und Problemgruppe}

Problematisierte soziale Gruppen treten in zwei Fassungen auf: als problematisiertes Kollektiv und als Problemgruppe. Wenn wir uns nicht nur die staatlichen Instanzen anschauen, sondern auch diejenigen, die es betrifft, haben wir zunächst den Fall, dass Menschen, die mit Problemzuschreibungen konfrontiert sind, diese ablehnen und sich dagegen wehren. Dies erfolgt immer in Bezug auf eine allgemeine Bevölkerung, der sich die Zugehörigen zurechnen. Wir haben es mit einem problematisierten Kollektiv zu tun. Es ist eine Kategorie im Umlauf, die als Sammelbegriff fungiert die Migrant*innen, die Muslime, die Rechten. Dieser Bezeichnung haften erste Problemzuschreibungen an, die öffentlichkeitswirksam thematisiert werden, wie der Fall des Populismus eindrücklich zeigt. Sie verlangen den Bezeichneten bereits einiges $\mathrm{ab}$, um die Anhaftungen abzuschütteln und das Problematische aus der Mitgliedschaftskategorie herauszuhalten (Negnal 2019b). Muslime sind gefordert, sich von Islamisten abzugrenzen. Neue Rechte versuchen Volksverhetzungen zu relativieren. Die Mitgliedschaftskategorie hat einen prekären Status, denn Zugeordnete können jederzeit mit den Problemzuschreibungen konfrontiert werden. ${ }^{4}$ Sie versuchen dies zu vermeiden. Goffman (1990, S. 115 ff.) hat die Techniken der Informationskontrolle eingehend beschrieben, mit denen sich die Akteur*innen von Stigma-Symbolen distanzieren bzw. dagegen vorgehen wollen.

Es gibt aber auch jene, die offensiv mit solchen Zuschreibungen umgehen, mehr noch, sie selbst mit hervorbringen. Dies ist nicht als Reaktion zu verstehen, sondern vielmehr als ein aktives Mitmachen an der Problematisierung. Hier haben wir es mit einer Problemgruppe zu tun. Es ist eine Mitgliedschaftskategorie, für die auch eine Bezeichnung im Umlauf ist, jedoch keine allgemeine wie beispielsweise Muslime, sondern eine konkrete, etwa „Nationalsozialistischer Untergrund“ (NSU) oder „Identitäre“. Zudem werden vielschichtige Gruppenbezüge hergestellt, die undurchsichtige Verhältnisse nahelegen. Es ist unklar, inwieweit es sich um einzelne Gruppierungen, Netzwerke, Clans, Organisierte Kriminalität oder autonom agierende Splittergruppen handelt. Daraus ergibt sich der Umstand, dass fehlendes Wissen zur Problemgruppe beklagt wird, weil es notwendig scheint, spezifisches Wissen zu dieser Gruppe anzuhäufen. Die Generierung von Wissen über die Problemgruppe geschieht vor allem vor dem Hintergrund der institutionellen Bearbeitung der Zugehörigen. Im Vergleich zum problematisierten Kollektiv wird bei der Problemgruppe eine Bearbeitbarkeit angenommen, die beim problematisierten Kollektiv diskriminieren und auch ausufern würde, weil sich die Zugeordneten immer auch der allgemeinen Bevölkerung zurechnen.

Im Unterschied zum problematisierten Kollektiv wird bei der Problemgruppe die Mitgliedschaftskategorie aktiv gewendet. Repräsentant*innen sind daran interessiert

\footnotetext{
${ }^{4}$ Siehe auch die Analyse von Ammann Dula (2019) zu Einwander*innen in der Schweiz.
} 
als problematisch erkannt zu werden. Sie kommunizieren dies über ihre Handlungen wie über Symbole, Farben und Gegenstände (beispielsweise die gelb-schwarzen Fahnen der Identitären, die Logos und Schriftzüge der Ultras im Fußball). Gleichzeitig wechseln Zugehörige auch zwischen den kategorialen Einheiten - dem problematisierten Kollektiv und der Problemgruppe - und empören sich über Verdachtskonstruktionen (etwa Identitäre, die auch Parteimitglieder der AFD sind). Solche multiplen Mitgliedschaften lassen Statusbestimmungen unscharf zurück. Auch dies erfordert von den Beteiligten einiges an Arbeit. Wie das gelingt, zeigt sich in den im Folgenden dargestellten Konstruktionsmechanismen.

\section{Der Produktionsprozess der Problemgruppe}

In meiner Forschung zu Problemgruppen im Strafvollzug habe ich untersucht, wie Inhaftierte, die als besonders brutal, unberechenbar und nicht mitarbeitsbereit gelten, zu einer sozialen Gruppe homogenisiert und als besonders qualifiziert werden (Negnal 2016, 2019a). Folgt man dem Labeling Approach, werden im Zuge der Konstruktion einer Problemgruppe Betroffene geschaffen, die über Selbst- und Fremdzuschreibungen ins gesellschaftliche Abseits geraten (u.a. Becker 2014; Quensel 1970; Karstedt 1975; Scheerer 1978). Als Randgruppe (Karstedt 1975; Fürstenberg 1965) würden sich die zugehörig Definierten in die Rolle als Stigmatisierte einfügen. Dies trifft insofern zu als Ultras, Identitäre oder Salafisten keinen gesellschaftlich anerkannten Status erreichen, was beispielsweise Gegendemonstrationen nahelegen, bei denen die allgemeine Bevölkerung Alarm schlägt. Allerdings vermeiden die Betreffenden es auch nicht, diskreditier- und sanktionierbar zu sein, obwohl sie von Ausgrenzungen betroffen sind, wie Ausstiegsprogramme proklamieren. Es geht ihnen aber auch nicht darum, Verbesserungen ihrer Situation voranzutreiben. So wollen Ultras ,ihre Mannschaft" unterstützen und das Spielereignis gestalten (Thalheim 2019), nicht die Fußallliga reformieren. Hier greift das Randgruppenkonzept demnach nicht. Wenn also weder allein Opfer- noch ausschließlich Täterkonstrukte adressierbar sind, wie entstehen und etablieren sich dann Problemgruppenzugehörige?

Goffman weist hier auf einen wichtigen Aspekt hin. Mit der Rahmung einer Situation stehen bestimmte Akteurskategorien zur Verfügung, denen Eigenschaften zugeschrieben werden (1990, S. 11f.). Problematisiert werden jene, die ,mit unserem Stereotyp von dem, was ein gegebener Typus von Individuum sein sollte, unvereinbar" sind (ebd., S. 12). Eine Eigenschaft kann demzufolge je nach Situation und antizipierter sozialer Zugehörigkeit der Situationsbeteiligten, als „normal“ oder als ,abweichend“ angesehen werden (Peters 2009, S. 61). Gleichzeitig sind Zuschreibungen nicht beliebig. Die Frage ist demnach, welche Akteurskategorien in Anschlag gebracht werden und welche sich in einer vorgefundenen Situation durchsetzen.

Wiederholte Auftritte der Beteiligten, die medial und vermittels staatlicher Beschäftigung der Verfolgungsbehörden eine Rollenkontinuität der Akteur*innen ermöglichen, tragen in erheblichem Maß dazu bei, dass sich Bilder von Ablehnenden und Gewalttätigen verfestigen. Die Gruppenzuschnitte entwickeln sich im ,ge- 


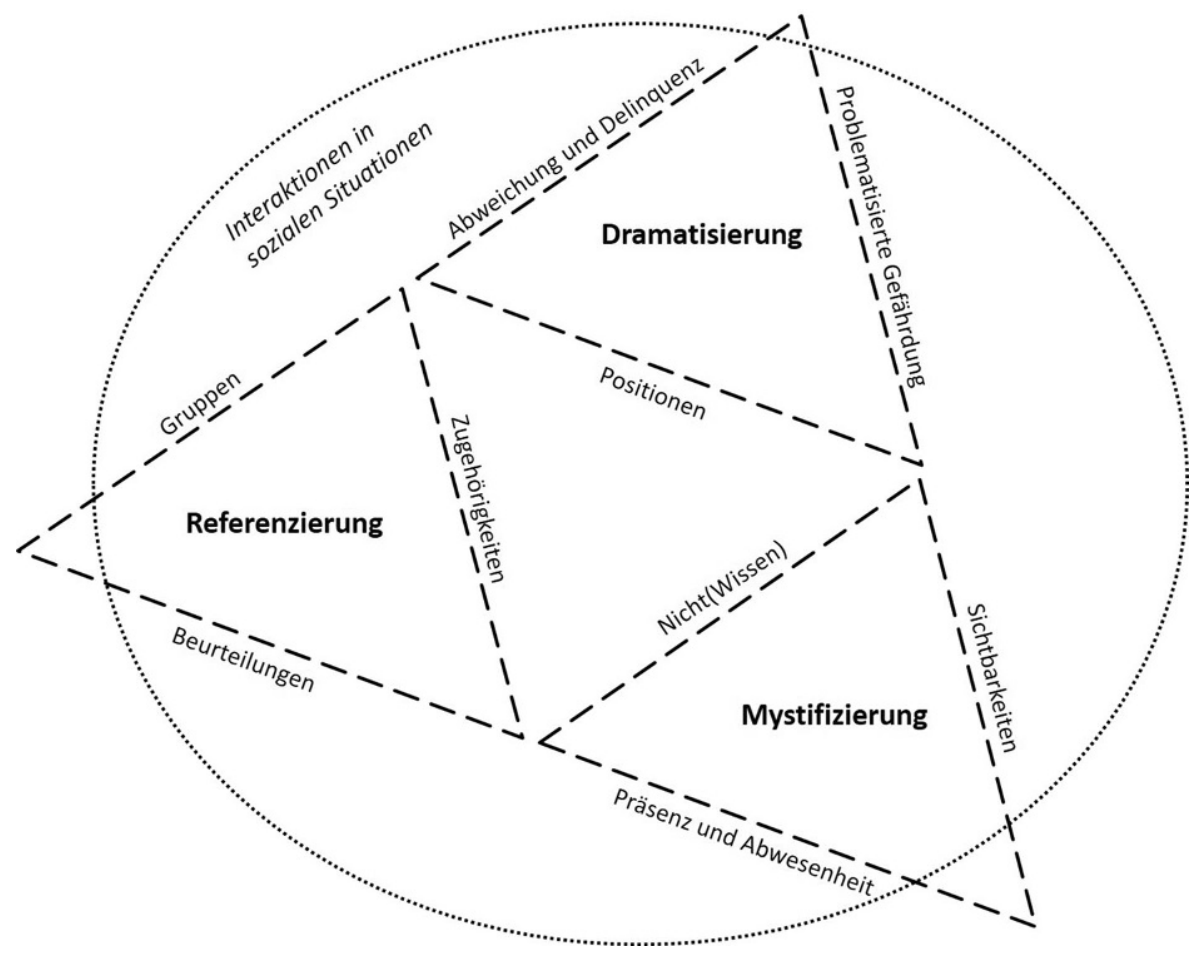

Abb. 1 Konstruktionselemente der Problemgruppe (vgl. Negnal 2016)

meinsamen" Abarbeiten am Problematischen. Dabei handelt es sich nicht nur um Fremdzuschreibungen, ebenso wenig lassen sich die Selbstzuschreibungen als problematisch nur als Reaktionen fassen. Es stellt sich vielmehr diese Frage: In welchen Situationen und Kontexten sind Menschen daran interessiert, als problematisch zu gelten? Wie und wo wird also die Mitgliedschaftskategorie relevant?

Eine Problemgruppe entsteht in drei miteinander verwobenen Prozessen: Referenzierung, Dramatisierung und Mystifizierung (Negnal 2016, S. 270 ff.). Um die verschiedenen Elemente zu charakterisieren, werde ich nun zunächst das jeweilige Konzept skizzieren, Beispiele aus meinen Forschungen anführen und jeweils Anschlüsse zu weiteren Problemgruppen liefern. Für einen Überblick sind die Kernelemente der Konstruktion von Problemgruppen in obiger Grafik gleichrangig aufgeführt. In der sozialen Situation gelangen die Phänomene in unterschiedlicher Gewichtung zur Geltung. (Abb. 1).

In den Prozesselementen der Referenzierung, Dramatisierung und Mystifizierung gehen Phänomene auf, die das Konstrukt Problemgruppe ausmachen. Die Referenzierung bezieht sich auf Zugehörigkeiten, Beurteilungen und Gruppen. Zur Dramatisierung braucht es Phänomene wie Abweichung und Delinquenz, Positionen und eine problematisierte Gefährdung. Die Mystifizierung verläuft über Phänomene von (Nicht)Wissen, Sichtbarkeiten sowie Präsenz und Abwesenheit. 


\subsection{Referenzierung}

Treten Akteur*innen in soziale Interaktionen, gestalten sie ihre Handlungen verstehbar und anschlussfähig. Dafür verweisen sie auf Personen, Ereignisse und andere soziale Situationen. Mit den Bezügen, den Referenzen, nehmen die Akteur*innen $\mathrm{Zu}$ - und Einordnungen vor. Die Referenzierung bezeichnet also das Herstellen dieser Bezüge. Die Verweise sind notwendig, um sich während des Geschehens in Beziehung zu anderen setzen zu können und so eine soziale Situation zu gestalten. Bei problematisierten sozialen Gruppen sind drei Typen von Referenzen ausschlaggebend: Bezüge zu Gruppen, Bezüge, die diffuse Zugehörigkeiten nahelegen und Bezüge durch (Verhaltens)Beurteilungen.

Gruppenbezüge werden beispielsweise hergestellt, wenn ein neu Inhaftierter versucht, einen Bezug zum „Russischen“ herzustellen - etwa indem er auf dem Freistundengelände Russisch spricht oder russischsprachige Gefangene benennt, die ihm bekannt seien. Solche Bezüge stellen eine Art Bewerbung dar, da sie die Zugehörigkeit zur Problemgruppe bekunden. Das nehmen die Repräsentant*innen der Problemgruppe zum Anlass, ein eigenes Regelwerk auf den Bewerber anzuwenden und Beurteilungen zu dessen Eignung und Funktionsfähigkeit vorzunehmen (Negnal 2016, S. 174 ff.). In Ansprachesituationen, in denen die Betreffenden sich selbst als Zugehörige bezeichnen oder von anderen so bezeichnet werden, bringen sie sich als jemand in Stellung, der im Zusammenhang mit einer Problemgruppe Beachtung finden sollte. Dies geschieht, indem bestimmte Begriffe oder Personennamen fallen gelassen oder Ereignisse angedeutet werden, um die eigene Zugehörigkeit zu diesem Ensemble zu bekunden. Hierbei handelt es sich keineswegs um Geheimwissen, sondern um Informationen, die im Diskurs um die Problemgruppe verfügbar sind.

Mit dem Kundtun der Zuordnungen setzen sich die Betreffenden zu Repräsentant*innen der Problemgruppe in Beziehung und umgekehrt. Es werden Teilhabemöglichkeiten an Gruppen signalisiert, die Unterstützung und Fürsorge bedeuten können und so einen Freiraum kreieren. Dies ist insbesondere im Kontext staatlicher Verfolgungsbehörden, wie im Strafvollzug, relevant. Eine Handlungsweise russischsprachiger Inhaftierter ist hier beispielsweise, sich jemandem zuzuwenden und Ansprechbarkeit zu signalisieren bzw. Schutz, Hilfe oder generell Aufmerksamkeit zu schenken. Damit wird dem Gegenüber Überlegenheit und ein Etabliertsein unter den Inhaftierten angezeigt, wodurch weitere Inhaftierte und auch das Personal aufmerksam auf den Betreffenden werden.

In der von mir beforschten Haftanstalt haben Personal und Inhaftierte immer wieder bestimmte Begriffe angebracht - so wurde in einer Abteilungsbesprechung über Gegenstände, die von Inhaftierten aus Brotteig hergestellt worden waren, als "Waffe" gesprochen - oder auf bestimmte Personen und Szenarien angespielt - etwa indem an eine Massenschlägerei auf dem Innenhof erinnert wurde, die zwei Jahre zurücklag. Mit solchen Referenzen eröffnen die Beteiligten den Bezugsrahmen einer "Gefährdung“. Dies gelingt ihnen in zweierlei Hinsicht. Zum einen legen die schlagwortartigen Referenzen - beispielsweise „Waffe“ oder tätowierte „Zwiebeltürme" - eine Situationsdefinition nahe, in die sich andere an der Situation Beteiligte leicht einbringen können. Als Mitglieder des situativen Ensembles bezeugen sie das Problematisierte, weil sie auf geteilte Erzählungen zurückgreifen. Die Bezüge, z.B. 
die Gewalttätigkeit, werden durch bestimmte Techniken, z.B. das Bezeichnen einer vermeintlichen Waffe oder das Aufwärmen von Geschichten, verwirklicht. Hier greift ein situativer Zug, denn eine Gefährdung wird zum vermeintlichen Handlungsproblem aller und ruft zur Aktivität auf. Zudem rangieren die Bezüge auf verschiedenen Ebenen - Referenzen zu Mitgefangenen, zur Ethnizität, zum eigenen Regelwerk, zu Geschichten über eine kriminelle Unterwelt. Sie gelangen jedoch gleichzeitig zur Geltung und suggerieren, dass es Zugehörigkeiten von Inhaftierten zu diversen Handlungsgruppen gibt.

Die Referenzierung bedeutet für Alltagssituationen, Abgrenzungen vorzunehmen und sich zu positionieren. Es bedeutet Gemeinsamkeiten herzustellen, Allianzen zu bilden und Konkurrenz zu verhandeln, sich also in situ sowohl als Person als auch als Repräsentant*in eines sozialen Zusammenhangs auf die Suche nach einem Platz im sozialen Gefüge zu begeben. Mit dem Kundtun von Zuordnungen und dem Sich-selbst-in-Beziehung-Setzen können Sozialbindungen angebahnt, erhalten und ausgeschlagen werden, weil Bezugspunkte im sozialen Gefüge als Appell an diverse Zugehörigkeiten gerichtet werden, die eine Verortung in der sozialen Situation ermöglichen.

Wenn bei einer Demonstration beispielsweise ein*e Journalist*in einen Teilnehmer nach seinem Anliegen befragt und dieser die Anfrage mit dem Ausspruch „Verräter“ zurückweist, dann stellt sich auch hier die Frage nach der Selbstzuordnung des Gefragten. Naheliegend wäre, dass er als Demonstrant ein Anliegen hat, das er kundtun möchte und dass er daher über eine Interviewanfrage dankbar sein würde. Mit seiner Antwort knüpft er jedoch an eine andere soziale Zugehörigkeit an, beispielsweise die der Identitären, die einen Kontakt mit der Presse ablehnt. Aus Sicht des Journalisten mag sich der Teilnehmende dadurch zwar disqualifizieren und als abweichend gelten, doch die Selbstzuordnung als Identitärer verschafft ihm eine andere Anerkennung. Er wird diese Zugehörigkeit nicht verschleiern, vielmehr wird er sie als Begründung für sein Verhalten anbieten und so aktiv das Bild des Abweichlers gestalten. Dieser Prozess hat also immer auch mit Positionen, einem Element der Dramatisierung, zu tun.

\subsection{Dramatisierung}

Positionierungen markieren Handlungsmacht bzw. -ohnmacht. Ein russischsprachiger Inhaftierter äußerte sich zum Beispiel über einen Mitgefangenen, der sich bei einer Zusammenkunft russischsprachiger Gefangener aus seiner Sicht falsch verhalten hatte, als er seinen Teller nach dem Essen nicht selbst abräumen wollte. Im Interview kommentiert der Gesprächspartner seine Beobachtung des Mitgefangenen mit dem Ausspruch: „Da dachte ich, du bist hier ganz falsch“. Er grenzt sich also von dem Mitgefangenen ab, wenn er die Zusammenkunft als Ereignis einer spezifischen Gruppierung einführt und damit die soziale Gruppe russischsprachiger Inhaftierter aufruft. Gleichzeitig deutet er das Regelwerk dieser Inhaftierten an und markiert sich als Mitglied mit einem Führungsanspruch, das über den Ausschluss des Mitgefangenen sinniert.

Oder eine Vollzugsmitarbeiterin beklagt in einer Besprechung, dass sie die körperliche Auseinandersetzung zwischen zwei Gruppierungen russischsprachiger In- 
haftierter nicht verhindern könnten und leitet dies damit ein, dass „,die Russen was planen würden“ (Feldnotizen). Die mit der Referenzierung angebahnten Zuordnungen werden gefestigt. Die Bedienstete macht klar, dass russischsprachige Inhaftierte in der Abteilung zu den Delinquenten gehören. Die Totalität, die eine Position bei problematisierten sozialen Gruppen andeutet, benötigt gleichzeitig Pauschalurteile. Positionen kaschieren daher auch Unwägbarkeiten und Ungenauigkeiten. Um dennoch eine haltbare Position einnehmen bzw. formulieren zu können, wird zum einen die problematisierte Gefährdung bemüht, zum anderen werden Positionen in für die Problemgruppe zur Verfügung stehenden Rollenkonzepten ${ }^{5}$ eingeübt, mit denen die Regeln fortlaufend aktualisiert werden.

Auch sog. Salafisten beziehen sich auf ein Regelwerk, das an ihre Auslegung des Glaubens gebunden wird. Hooligans beziehen sich bei ihren Treffen auf ein Regelwerk, das sich an Sportrichtlinien orientiert. Diese Regeln sind nicht nachzulesen. Sie kursieren im Mündlichen und erfahren ihre Geltung in der Handlung.

Die problematisierte Gefährdung wird zum Situationselement, wenn eine Gefährdungslage für sich selbst oder für andere thematisiert wird. Problematisiert wird diese Lage durch Unzulänglichkeiten und Ungewissheiten. Der Inhaftierte, der sich über seinen Mitgefangenen äußert, unterstellt ihm weitere Regelverstöße, die gemeinsame Aktionen der sog. „Gesetzrussen“ (Negnal 2016) gefährden könnten. Aber auch Personalmitglieder sprechen von „Waffen“, welche russischsprachige Gefangene besäßen, ohne z. B. zu präzisieren, wer diese Waffen habe oder um welche Art Waffe es sich handle. Ebenso drohen russischsprachige Inhaftierte ihren Mitgefangenen damit „,durchzudrehen“, was dazu führt, dass die Mitgefangenen ihnen erteilte Aufgaben erfüllen und sich zurückziehen, womit die Ordnung in dieser Situation wiederhergestellt wird. Es geht hierbei um Zuschreibungen problematischen Verhaltens und problematisierter Eigenschaften, wie Unberechenbarkeit oder NichtIntegrierbarkeit (Negnal 2016, S. 104 f.). Ein gescheitertes Gespräch bringt eine Sozialarbeiterin dazu, russischsprachige Inhaftierte generell als „Wilde“ (ebd., S. 105) zu bezeichnen. Andere monieren, keine Chance zu haben, überhaupt irgendeinen Zugang zu ihnen zu finden (Feldnotizen), indem beispielsweise betont wird, dass sich das Potenzial russischsprachiger Inhaftierter im Umgang mit dem Vollzugspersonal zu Extremfällen ausweiten kann. So kann die Belastung im eigenen Arbeitsalltag thematisiert, Berichte können geschrieben und weitere Ressourcen mobilisiert werden, etwa indem neue Stellen eingerichtet werden. Hierzu tragen auch kleinere Gesten der Inhaftierten bei, wie etwa eine zuvor angeregte Gesprächssituation durch Schweigen zu unterbrechen, um Spannungen aufzubauen und auch um zu demonstrieren, solche Spannungen ohne Weiteres aushalten zu können. Inhaftierte können voreinander von Bedrohungen durch russischsprachige Gefangene sprechen und andere als die Anstaltsregeln thematisieren. Damit werden russischsprachige Inhaftierte als Akteure erst greifbar, denn es ist ihr Regelwerk, das eine Drohkulisse

\footnotetext{
5 Etwa das Konzept des „Aufsichtsrats“, mit dem kontrolliert, organisiert und Fürsorge betrieben wird; das Konzept des „Übermittlers“, mit dem Informationen, Aufträge und Güter übermittelt werden, indem sich Inhaftierte auf den „Kampf für ein System“ (Negnal 2016, S. 170) beziehen, dem sich viele Weitere zugehörig fühlen; und das des „Ergebenen“, der sich dem Regelwerk unterwirft und anderen folgt sowie Maßregeln und Sanktionen über sich ergehen lässt (ebd., S. 172).
} 
aufbaut (ebd., S. 217 ff.), und es sind ihre Strategien zu einer überlegenen Haltung, die eine Gefährdung für andere zumindest nicht auszuschließen scheinen.

Mit den Darstellungen von Offensivität und Brutalität russischsprachiger Inhaftierter erscheint das eigene Handeln als nicht Problematisierter folgerichtig und appelliert zugleich an weitere Akteur*innen, sich ebenso zu beteiligen, um dieser Gefahr auszuweichen oder ihr etwas entgegenzusetzen. Die von einer*einem Akteur*in ins Spiel gebrachte Definition als gefährliche Situation soll durchgesetzt werden. Das Dramatisieren hinsichtlich der problematisierten Gefährdung ist der Versuch von Akteur*innen, andere für eigene Belange zu gewinnen und sie für die Legitimation der eigenen Rolle und den hiermit verbundenen Aufgaben zu nutzen. Damit rekrutiert sich ein*e Akteur*in also ein Ensemble, mit dem sie*er gemeinsam die für die Situationsdefinition notwendigen Elemente aktualisiert. So wird nachvollziehbar, dass von „Waffen“ und „Russen“ gesprochen wird, ohne eine Intervention zu beschließen oder selbst tätig zu werden. Mit der problematisierten Gefährdung kann die eigene Handlungsohnmacht thematisiert werden, ohne jedoch den eigenen Status zu riskieren.

Die Dramatisierung kennzeichnet das Betonen des eigenen Agierens. Das von Erving Goffman entlehnte Konzept der dramatischen Gestaltung als Inszenierung dessen, was man eigentlich gerade tut (Goffman 1973b, S. 31 ff.), liefert den anderen an einer Situation Beteiligten Beweise für die eigene Fähigkeit, die „Hauptaufgabe der Rolle“ (ebd., S. 31), die man gerade übernommen hat, zu erfüllen. Die dramatische Gestaltung hebt also die eingenommene Rolle vor anderen hervor. Dadurch können Aufmerksamkeiten gebündelt werden und wenig beachtete Aspekte des eigenen Tuns erfahren Interesse. Wenn Ereignisse, Handlungen, Personen, zugeschriebene Eigenschaften und auch Positionen betont werden, können die mit der Referenzierung angebotenen Zugehörigkeiten (und auch Abgrenzungen) etabliert werden, indem man die eigene Rolle vor anderen illustriert. Zugleich kann mit dem Betonen kenntlich gemacht werden, welche Rollenerwartungen andere Akteur*innen an die Betreffenden herantragen. So können Zuschreibungen unterstrichen und gefestigt werden. Dies geschieht hinsichtlich von Positionen, einer problematisierten Gefährdung sowie von Abweichung und strafrechtlich relevanter Delinquenz.

Abweichung und Delinquenz sind Aspekte der Konstruktion, um Normen zu verdeutlichen, an die sich die Problemgruppenzugehörigen vermeintlich nicht halten oder anpassen. Da subkulturelle Gruppen oder Gewalthandeln verschiedenen Szenen als üblich angetragen werden, werden im Zuge der Dramatisierung besonders eindrückliche Fälle betont, um die Gefährlichkeit der Problematisierten darzustellen und ihre Abweichung wie Delinquenz zu unterstreichen. Damit gelingt es aus Sicht der Problembearbeiter*innen, die Relevanz der eigenen Tätigkeit hervorzuheben und sich gleichzeitig als abgeklärt und handlungsmächtig zu präsentieren.

Dramatisierungen strukturieren Interaktionen. In den Kategorien Abweichung, problematisierte Gefährdung und soziale Position können vorgenommene Zuordnungen etabliert werden. Manche Elemente kommen erst zur Geltung, wenn sie in Übertreibungen auftauchen. Das Betonen lässt den*die Akteur*in handlungsmächtig erscheinen, ohne dabei explizit zu machen, worauf sich diese Position gründet. Was sich so in der Referenzierung anbahnt, kann mit der Dramatisierung ausgebaut 
werden, indem das eigene Tun unterstrichen und auch weitere Ensemblemitglieder rekrutiert werden, sich an der initiierten Definition zu beteiligen.

\subsection{Mystifizierung}

Während bestimmte Referenzen angebracht werden, die die Problematisierung einer sozialen Gruppe anstiften und das Betonen in Bezug auf die Problemgruppe die eigenen Verwicklungen unterstreicht, scheinen andere Aspekte hingegen kaum greifbar zu sein. Ein Regelwerk, das mit geltendem Recht unvereinbar scheint, das Zur-Schau-Stellen von Gegenständen, die auf abweichendes Verhalten schließen lassen oder das wiederholte Auftauchen von Leuten in „heiklen Situationen“ belegen eine vermeintliche Mitgliedschaft.

Die Mystifizierung als drittes Element in der Konstruktion einer Problemgruppe hat mit diesen Unwägbarkeiten zu tun. Goffman hat in „Wir alle spielen Theater“ (1973b) Darstellungen von Akteur*innen beschrieben und hier eine Strategie herausgearbeitet. Die Mystifikation beschreibt er als Strategie der „Selbstverhüllung“ (1973b, S. 63) mit dem Effekt, eine soziale Distanz zwischen Darstellenden und Publikum zu erreichen. Dieser Effekt muss allerdings erst erarbeitet werden. Problematisierte soziale Gruppen sind von einem Arsenal staatlicher wie nicht-staatlicher Akteur*innen umgeben, die mit den oder gegen die Zugehörigen arbeiten (wollen). Zugehörige problematisierter sozialer Gruppen sind jedoch nicht zu jeder Zeit in gleicher Intensität sichtbar. Die Zugriffs- und Lenkungsversuche werden daher von den Beteiligten kanalisiert und die jeweils ,,anderen“ so auf Distanz gehalten. Die soziale Distanz hat mit den Versuchen zu tun, die Wahrnehmungen der anderen zu lenken, z. B. durch den Rückzug aus der Situation, das Angebot bestimmter Informationen oder die Verweigerung, Anweisungen zu folgen. Eindrückliche Beispiele hierfür liefern auch die Blick-, Rede- und Zeigepolitiken russischsprachiger Inhaftierter. Indem verbotene Gegenstände so platziert werden, dass Bedienstete sie „finden“ oder in einem vorgetäuschten Wutausbruch Gegenstände zerstört werden, kann die Aufmerksamkeit der Vollzugsmitarbeiter*innen von anderen Verstecken oder Gegenständen abgelenkt werden. Auch hier wird das Interesse des Personals auf eine bestimmte Abteilung gelenkt und die Inhaftierten schaffen es, eine sonst von Unsicherheit geprägte Lage, wie sie die Durchsuchungspraxis in einer Haftabteilung aufgrund von Stichprobenkontrollen entstehen lässt, für einen Moment zu kontrollieren und zu durchbrechen.

Repräsentant*innen einer Problemgruppe bleiben präsent, weil ihnen Ereignisse oder Momente, in denen beruhigt geglaubte Lagen neu entfacht werden, zugeschrieben werden können. So bleiben die Positionen im sozialen Gefüge erhalten, auch wenn es beispielsweise aktuell keine Fälle von Abweichung und Delinquenz zu verzeichnen gibt. Dies betrifft zum Beispiel auch regelmäßig die Aufklärungsveranstaltungen von Polizei und Verfassungsschutz über Linksextreme (Scheffer et al. 2017). Hierfür mitverantwortlich sind die Dramatisierungen, also Darstellungen in so massiver Weise, dass sie als Eindruck der Situation auch bei eigener Abwesenheit bewahrt bleiben. Mit Blick auf russischsprachige Inhaftierte betrifft das körperliche Auseinandersetzungen zwischen Inhaftierten ebenso wie intensive Konfrontationen mit dem Personal. Das heißt, Übertreibungen in Form von Dramatisierungen gebie- 
ten möglichen Zweifeln Nicht-Zugehöriger Einhalt, eine soziale Gruppe sei nicht mehr problematisch. Damit wird eine etwaige aufkommende Skepsis zur Existenz von bestimmten Gruppen ausgeräumt. Die Mystifizierung durch Präsenz und Abwesenheit ermöglicht, die soziale Distanz stabil zu halten. Mit Andeutungen, faktisch anmutenden Tatsachen und artikuliertem Misstrauen wird die Erkennbarkeit von Personen und Verhaltensweisen manifestiert.

Das Arbeiten an der sozialen Distanz über das Generieren von Verdachtsmomenten hat jedoch auch eine Kehrseite. Ein Fehlverhalten kann jederzeit unterstellt werden, unabhängig von seiner Nachweisbarkeit. So werden die Beziehungen zwischen den Beteiligten gefährdet, wenn beispielsweise von einer Inhaftiertengruppe ein Ausschlussverfahren eines Mitgefangen in Gang gebracht wird, obwohl die angenommene Regelverletzung nicht nachweisbar ist (Negnal 2016, S. 206ff.). Dass der Betreffende sich tadelhaft verhalten hat, scheint für die Anklagenden nicht in Frage zu stehen, nicht zuletzt, weil vorherige Dramatisierungen nun Anhaltspunkte liefern. So laufen soziale Beziehungen stets Gefahr, destabilisiert zu werden, während sich die soziale Distanz festigt.

Die Zugriffsversuche auf Problemgruppenzugehörige betreffen auch das (Nicht) Wissen zu dieser sozialen Gruppe. In meinem aktuellen Forschungsprojekt zu den Widerständigkeiten gegen eine Radikalisierung ${ }^{6}$ kämpfen Sozialarbeitende damit, zu wenig über „den Islam“ zu wissen, um eine Radikalisierung ihrer Klient*innen erkennen zu können. Dennoch sind sie gefordert, dies in ihrer Arbeit zu berücksichtigen. Dieses Wissen wird exklusiv, wie sich beim Umgang mit Informationen zeigt. Es wird versucht, einen Kreis von Informierten abzustecken, sei es mittels kontraktförmiger Erklärungen wie zur Schweigepflicht von Anstaltspersonal und Trainer*innen in der Deradikalisierungsarbeit oder bei der Aufnahme von russischsprachigen Inhaftierten in Gruppierungen. Für das Streuen und Bewahren von Informationen werden bei den Interessent*innen mögliche Bedarfe, die sie empfänglich machen könnten für eine ungewollte Informationsweitergabe, ihre Erfahrungen im institutionellen Austausch und ihre Wissensbereiche, die gewinnbringend sein könnten, eingeschätzt (vgl. Negnal 2016, S. 178 ff.). Mit dem Spiel von Wissen und Nichtwissen können Inhaftierte gegenüber dem Personal für Ungewissheit darüber sorgen, ob sie zu einer so genannten subkulturellen Gruppierung gehören oder nicht. Dabei finden auch Ungewissheiten, Fehlinformationen und Irrglaube in der Darstellung Berücksichtigung.

Die Inszenierungen von Problemgruppenmitgliedern wie ihren Bearbeiter*innen zeichnen sich dadurch aus, dass sie vor dem jeweiligen Gegenüber Wissen darstellen. Dabei werden jedoch keine Erklärungen oder Beschreibungen abgegeben, sondern es wird mit überschriftartigen Aufzählungen und Andeutungen gearbeitet, die die Beteiligten als Träger*innen exklusiven Wissens ausweisen. Dies zeigt sich etwa in den Trainings, die die in der Deradikalisierungsarbeit Tätigen den Vollzugsmitarbeitenden anbieten. So bleibt für viele Vollzugsmitarbeiter*innen unklar, wie Mitglieder von Problemgruppen erkannt werden können. Dafür scheint das Spezialwissen über die Problematisierten zu komplex, weshalb man mit Beispielfällen operiert, in denen

\footnotetext{
${ }^{6}$ RESIST wird vom Bundesministerium für Familie, Senioren, Frauen und Jugend gefördert. Informationen unter https://www.bildung.uni-siegen.de/mitarbeiter/negnal/resist/index.html. Zugriff am 02.09.2020.
} 
so oder so verfahren worden sei, um sich selbst in der Auslegungspraxis orientieren zu können. So entsteht die Position ,eingeweihter Expert*innen“, die Symbole (Gegenstände und Personen) benennen und sie in Narrative, vor allem in Form von Erzählungen, verwickeln. In der Konsequenz entsteht eine asymmetrische Ordnung der Situationsteilnehmer*innen mit dem Effekt, dass durch das Außenvorlassen Freiräume entstehen, woraus die Betreffenden ein erweitertes Handlungsrepertoire schöpfen können (Goffman 1973b, S. 63). Genau in diesem Ringen um Zugriff und entsprechenden Entzugs- bzw. Lenkungsversuchen entsteht mit der Mystifizierung die Problemgruppe als gefährliche Gruppe, die kollektiv gewusst wird, weil sie in Geschichten zirkuliert und in Aufführungen in Szene gesetzt wird.

Dies geschieht etwa, wenn nicht erlaubte Gegenstände in einer Wohngruppe in Jugendhaft (zum Drogenkonsum oder Mobiltelefone) Restriktionen nahelegen, die jedoch nicht eindeutig einer Person zuzuordnen und auf direktem Wege nicht durchzusetzen sind. Frustration und Unsicherheiten im Umgang mit den problematisierten Inhaftierten werden von diesen geschürt. Oft wird mit Andeutungen gearbeitet. So bleibt im Verborgenen, wie eine Gefährdung besteht und Zugehörigkeiten erscheinen undurchsichtig. Sie generieren Verdachtsmomente. Für die Konstruktion einer Problemgruppe bedeutet dieses Element, dass ein widersprüchliches Bild aufgerufen werden kann, das hinsichtlich der Zuschreibungen als gefährlich oder brutal konkret erscheint, aber in den Ausprägungen und in der Bestimmung der Zugehörigen vage bleibt.

Dabei erhält die Referenzierung die verschiedenen sozialen Gruppen, weil sie wiederkehrende Zuordnungen vorschlägt und Ensemblemitglieder rekrutiert, sich hinsichtlich der Problematisierung zu engagieren. Mit der Dramatisierung wird die Mitgliedschaft in einer Problemgruppe eindrücklich vorgestellt, indem die problematisierte Gefährdung zum zentralen Kriterium erhoben wird. Damit rücken auch diejenigen ins Visier, die noch nicht zur Problemgruppe gehören. Sie gelten als gefährdet und werden daher präventiv ,,unterstützt“. Das findet sich vor allem in der Deradikalisierungsarbeit. Die Mystifizierung unterfüttert die Dramatisierungen. Mit dem Arbeiten an einer sozialen Distanz wird es leichter, Mythen in Geschichten zu entwerfen. Die Betreffenden wiederum nutzen dies für ihre Handlungsfähigkeit, denn je weiter die Mythen zirkulieren und je extremer die Darstellungen in den Geschichten werden, desto größer sind in der Regel auch die Vorsicht und die Skepsis, mit denen den Betreffenden begegnet wird. Das wiederum ist hilfreich für Aktionen konkreter Gruppierungen, die die Distanz zu Außenstehenden aktualisieren und damit der Kohäsion der Mitglieder Vorschub leisten können. Mystifizierungen unterstützen schablonenartige Konturen als Rahmungswissen von Situationen, weil sie mit dem Arbeiten an der sozialen Distanz einhergehen.

\section{Das Werden sozialer Gruppen im Verhältnis zueinander betrachten - die Weiterentwicklung der Soziologie sozialer Probleme und sozialer Kontrolle}

Die Problemgruppe wird öffentlichkeitswirksam im gemeinsamen Abarbeiten am Problematischen hergestellt. Mit der öffentlichen Thematisierung (Blumer 1971) er- 
folgt immer auch ein Bezug nach Außen, der die Grenze zwischen Allgemeinheit und Problemgruppe aktualisiert, indem abweichendes Verhalten zur Verhandlungssache wird. In seiner wissenssoziologischen Formulierung skizziert Schetsche, dass soziale Akteur*innen Forderungen an staatliche oder nicht-staatliche Instanzen erheben, weil sie einen Verstoß gegen in der Gesellschaft dominierende Werte wahrnehmen, die Existenz von unschuldig Geschädigten beklagen und die Idee besteht, dass Abhilfe möglich sowie die Gesellschaft moralisch dazu verpflichtet ist, die kritisierten Zustände zu ändern (2001, S. 37). Dies lässt sich schärfen, wenn wir die Frage stellen, worauf sich das abweichende Verhalten bezieht. Die Problematisierung entfaltet hier kollektive Zugkräfte in dreierlei Hinsicht:

\section{Das abweichende Verhalten erscheint in organisiertem Ausmaß und legt ein} Gruppenphänomen nahe Den einer sozialen Gruppe Zugeordneten wird ein gewisses Maß an Engagement zugestanden - den Ultras die Anteilnahme beim Spiel ihrer Mannschaft, islamischen Vereinigungen die Religionsausübung und den Rechten und Linken die Auffassungen zum Staatswesen. Etwas ist nicht problematisch, weil es gegen gesellschaftlich dominierende Werte verstößt, wie Schetsche (2001, S. 38) ausführt, und auch nicht wenn ein Übermaß als abweichendes Verhalten Einzelner deklariert wird. Hier verfügen staatliche Instanzen über ausgreifende Bearbeitungskapazitäten, etwa in der Jugendhilfe und Justiz. Das Übermaß an Engagement wird jedoch mit sozialen Gruppen in Verbindung gebracht. Es wird also keine kategoriale, sondern eine relationale - auf Gruppen bezogene - Zugehörigkeit hergestellt. Mit der Unterstellung von Gruppierungen erhält das Übermaß an Engagement eine Organisiertheit, die staatliche Organe qua Auftrag anspricht, tätig zu werden, um die Ordnung zu erhalten. Soziale Gruppen werden zum Gegenstand von Problematisierungen.

Eine bedrohte Allgemeinheit zieht Grenzen Die Konstruktionen sind von einer Gefahren- und Drohkulisse geprägt, die, wie im Fall der russischsprachigen Inhaftierten in einer Jugendhaftanstalt oder vermeintlich radikalisierten Personen, ein Wissen über die soziale Gruppe erst erforderlich werden lässt. Dass die Mitarbeiter*innen staatlicher Instanzen zu wenig über die jeweils aktuell formierte Problemgruppe wissen, um sodann als Expert*innen ihre Klientel bearbeiten zu können, beklagen sie. Dies hängt mit der Abgrenzung, also der Markierung einer Differenz zwischen dem akzeptierten Engagement und dem zur Empörung aufrufenden Übermaß zusammen. Die Problemgruppe konstituiert sich ja gerade über mystifizierende Elemente, die zwischen den Akteur*innen eine soziale Distanz herstellen. Dadurch werden die Beteiligten zu Repräsentant*innen der jeweiligen sozialen Gruppe. Zugehörige einer vermeintlich gesellschaftlichen Allgemeinheit sehen das gesellschaftliche Gefüge durch die Präsenz der Problemgruppe gefährdet. Sie fühlen sich beispielsweise ,im eigenen Viertel fremd“ oder durch Hooligans und Neonazis während einer Demonstration in der Innenstadt bedroht, womit die Forderung an die Verantwortlichen sowohl in den staatlichen Instanzen als auch in den - so scheint es - ermöglichenden Kontexten einhergeht, tätig zu werden. Die Polizei soll gegen Krawallmacher vorgehen, islamische Vereinigungen sollen Aufklärungsarbeit leis- 
ten. Insofern sollen professionelle Bearbeiter*innen und problematisiertes Kollektiv die Problemgruppe einhegen.

\section{Zur Bearbeitung der Problemgruppe braucht es Kontakt zu ihren Repräsen-} tant*innen, der jedoch als höchst schwierig gilt. Die mit der Abgrenzung unterstrichene soziale Distanz erweist sich als Fallstrick im Zugang zur Problemgruppe. Teil des Engagements der Problemgruppenmitglieder ist es, sich auf eine andere, den Ausgeschlossenen übergeordnete Instanz, ein Regelwerk in Form von Gesetzen, Geboten und Rechten, mit dem das eigene Handeln legitimiert wird, zu beziehen. Das Handeln der jeweils Nicht-Zugehörigen erscheint entsprechend als nicht legitim und wird als abweichend kategorisiert. Linksautonome beklagen die Polizeigewalt bei Demonstrationen. Krawalle zeigen, dass es eben auch anders geht und die Polizei nicht die umfassende Hoheit besitzt. Die Polizist*innen wiederum beklagen den Widerstand und das Gewalthandeln der von ihnen als Krawallmacher Bezeichneten. So begründet sich das Verhältnis zwischen Zugehörigen und Nicht-Zugehörigen in einer Gegnerschaft und konzipiert ihre jeweiligen Mitglieder als Repräsentant*innen der gegnerischen Lager. Problemgruppen sind keine staatlich eingerichteten Phänomene. Sie sind gesellschaftlich verankert, weil sie im Miteinander von Allgemeinheit, problematisiertem Kollektiv und Problemgruppe entstehen und aktualisiert werden.

Mit Blick auf soziale Kontrolle und Hilfe erfüllen die Gruppierungen eine Funktion. Mit der Bezeichnung von Akteursgruppen werden soziale Probleme benennbar, die zwar im Miteinander entstehen, jedoch auf vermeintlich konkrete Gruppierungen projiziert werden können. Die Betreffenden wechseln: zum Beispiel von männlichen Spätaussiedlern (Negnal 2016) zu Nordafrikanern (Gruhlich 2019) oder Sinti und Roma (Scherr und Sachs 2019), das problematisierte Kollektiv der Einwander*innen jedoch kann in unregelmäßigen Abständen auf das politische Parkett geführt werden (Negnal 2016). Die Kehrseite dieser Benennung besteht in der Abgrenzung, wenn sich Gesellschaft und Staat den Problematisierten gegenübersehen. Ethnisierungen und Rassismus treten ihre Dienste dann als Begleiterscheinungen im Zuge der Abgrenzungen zur definierten Problemgruppe an. Mit der Etablierung einer Problemgruppe formiert sich eine vermeintlich gesellschaftliche Einheit gegenüber einer anderen. Zugehörige zu den jeweiligen Parteien werden im Diskurs unter anderem über ethnische, religiöse, politische und milieuspezifische Bezüge hergestellt, die sich in der konkreten Interaktion aus Devianz- und Desintegrationszuschreibungen speisen. Mit der Dramatisierung kann das Handeln der anderen skandalisiert werden, womit das eigene Tun als normativ ,richtig“ bewertet wird. Soziales Handeln im Zuge der Mystifizierung unterfüttert das Arbeiten an der sozialen Distanz, womit Ausgrenzungen und fehlende Zugänge thematisiert werden können, ohne den eigenen Status als legitimes Mitglied einer gesellschaftlichen Einheit zu gefährden (Negnal 2016).

Das Herstellen einer Gefahren- und Drohkulisse ist ein Element der Dramatisierung mit dem Effekt, die Ordnung in einer sozialen Situation wiederherzustellen. Insofern können Handlungsressourcen mobilisiert werden. Selbstzuschreibungen als gefährlich oder unberechenbar betonen auch die Exklusivität der Zugehörigen, zum einen, weil Selbststigmatisierungen Gemeinsamkeiten stiften können und andere Akteur*innen damit ausschließen. Zum anderen bedeutet der Ausschluss Anderer die 
Markierung einer Differenz. Das Sich-Anders-Verhalten konterkariert das Gleichmachen der Zugehörigen einer sozialen Gruppe und bedeutet keinesfalls nur eine Umgangsstrategie mit der institutionellen Übergriffigkeit, die mit der Problematisierung einhergeht. Die Markierung von Differenzen ist Teil der fortwährenden Positionierungen im Hinblick auf gesellschaftlich verortete Zugehörigkeiten.

Um zusammenzufassen: Der hier vorgestellte Ansatz, die Problematisierung sozialer Gruppen als kategoriale Verdichtung zu verstehen, ermöglicht eine Weiterentwicklung der für die Soziologie sozialer Probleme und sozialer Kontrolle prägenden konstruktivistischen Perspektive. Kennzeichnend sind interaktionistische Zugänge und damit einhergehend eine Prozessorientierung. Dies möchte ich abschließend in drei Aspekten pointieren.

Interaktionen ernst zu nehmen heißt, den Blick auf die akteurs- und situationsspezifischen Wechselwirkungen zu richten. Das Herstellen eines sozialen Problems ist ein Produktionsprozess, in dessen Zügen soziale Gruppen kreiert werden. Daher gilt es, Akteur*innen und ihre Beiträge in den Blick zu nehmen (1). Wenngleich der Konstruktivismus betont, dass soziale Probleme nicht unabhängig von den beteiligten Akteur*innen analysiert werden können, so gehen theoretische, methodologische oder disziplinenpolitische Positionen doch von kollektiven Akteur*innen aus, die ein Problemmuster formulieren (Schetsche 2014; Albrecht 2001), durch die dann Mechanismen sozialer Kontrolle sichtbar werden (Peters 2000). Kollektive Akteur*innen formieren sich jedoch erst in sozialen Situationen. Daraus folgt, dass die Beteiligten und ihre Beiträge in den Blick genommen werden müssen. Es stellen sich dann Fragen danach, wie die Beteiligungen gestaltet werden und welche Formen sie annehmen. Es sind die von Akteur*innen fortlaufend bewerkstelligten Situationen, die Prozesse freilegen, die bei der Problematisierung eine Rolle spielen.

Dass soziale Gruppen selbst zum Phänomen und damit zum Gegenstand für die Soziologie sozialer Probleme und sozialer Kontrolle werden, ist durchaus in den Handbüchern zu sozialen Problemen zu finden, z. B. bei Weis (1999), der ,rechte Rowdies“ als gewalthaftes Phänomen beschreibt, Naplava (2008), der „Intensivtäter" als Konstruktionsleistung polizeilicher Akteur*innen fasst, oder bei Stallberg (2008), der am Beispiel von religiösen Russlanddeutschen Integrationserwartungen nachzeichnet. Doch werden an ihnen andere Phänomene rekonstruiert. Die gesellschaftliche Kategorisierungsarbeit an sozialen Gruppen ist erst 2017 in einer Tagung der Sektion der DGS unter dem Titel „Problem- und Risikogruppen in Staat und Gesellschaft" thematisiert worden. ${ }^{7}$

An diesen Fällen lässt sich die Forderung nach der Berücksichtigung von Akteur*innen und ihren Beiträgen ganz grundsätzlich einlösen, denn sowohl das Soziale am Problem wird analysiert - die Beteiligungen an der Problematisierung als auch das Problematische wird expliziert: Die Problemgruppe erscheint als gefährliche Gruppe, die ein Sicherheitsrisiko für eine gesellschaftliche Allgemeinheit bedeuten würde. An den Personenkategorien brechen sich Problemzuschreibungen und ermöglichen so die Personifizierung sozialer Probleme. Implizit bleibt dabei

\footnotetext{
7 Auf die theoretischen Perspektiven und Fallstudien, u.a. zur Problematisierung von Einwander*innen (Gottschalk und Tepeli 2019; Scherr und Sachs 2019; Ammann Dula 2019), Fußballfans (Keysers et al. 2019) oder Homosexuellen (Lautmann 2019), wird bei Negnal (2019b) näher eingegangen.
} 
die Annahme, dass die Problematisierten allein betroffen sind, und in diesem Status eine gesellschaftliche Minderheit bilden.

In der Problematisierung sind diverse Züge erforderlich, damit eine soziale Gruppe zum Problem werden kann. Diese Züge ins analytische Blickfeld zu bekommen, heißt, den Fokus auf das Ringen um das vermeintlich Problematische scharf zu stellen. Daraus folgt, nicht die Betroffenen (und ihre Bearbeiter*innen) zu betrachten, denn dies unterstreicht den kategorialen Charakter der Betroffenen in ihrem Status als Problemgruppe oder auch als prekäre Gruppe und entwirft sie als eine gesellschaftliche Minderheit von Abweichenden. Das gemeinsame Herstellen von Problematisierten erfordert vielmehr eine Perspektivenverschiebung auf die Allgemeinheit. Wenn sich ein Problemdiskurs institutionalisiert, also bestimmte Problemdefinitionen sich setzen, während andere verblassen, ist dies eine Angelegenheit diverser Beteiligter und diese Verwobenheit gilt es, analytisch fassbar zu machen. Daher appelliere ich: Weg von den Betroffenen hin zu den Beteiligten (2)! Die Beteiligten setzen Zugehörigkeiten in Szene und schreiben sie anderen zu. Das muss im gesellschaftlichen Gefüge und Ringen miteinander zwischen Allgemeinheit und problematisiertem Kollektiv und Problemgruppe verstanden werden.

Hierzu sind staatliche Instanzen inklusive der Wissenschaft einzubeziehen (am ehesten bei Wehrheim 2018 formuliert). So stellt sich nicht die Frage, ob die Soziologie soziale Probleme benennen oder ,nur rekonstruieren“ kann, was die Akteur*innen ,selbst als relevant erachten“ (Groenemeyer 2001a, S. 15), denn Soziolog*innen sind immer Beobachter*innen und Teilnehmende im Prozess der Problematisierung. Dass Wissenschaftler*innen zur Benennung und Reproduktion sozialer Probleme beitragen, ist unbestritten (Hess 1993; Dollinger 2010; Wehrheim 2018). Doch wie wird das möglich? Wenngleich sie sich auch für Entproblematisierungen einsetzen, sind Wissenschaftler*innen zumeist Teil der Allgemeinheit und benennen das Problematisierte, zu dem sie selbst nicht nur eine forschungs-, sondern eben auch eine ethnomethodische Distanz haben, von dieser Warte aus. Die Politik sozialer Probleme zeigt sich eben nicht allein darin, dass zumeist staatliche Einrichtungen sich den konstatierten Problemen widmen, sondern auch darin, dass die Allgemeinheit an der Institutionalisierung, also der Setzung bestimmter Problemdefinitionen mitwirkt. Wenn sich ein Problemdiskurs institutionalisiert, also einigen Definitionen des Problems Vorrang vor anderen eingeräumt wird, wirkt es auf die Beteiligten zurück.

Insbesondere sind hier die Verschränkungen von sozialen Problemen und sozialer Kontrolle zu beachten (3). Problematisierungen und Kontrollaspekte sind miteinander verwoben. Dieser Verschränkung trägt der Ansatz der Problemgruppe als kategorialer Verdichtung Rechnung, denn die Problematisierung sozialer Phänomene - so auch sozialer Gruppen - gibt Anlass tätig zu werden, und gleichzeitig gilt: Wo soziale Kontrolle greift, können Abgrenzungsbestrebungen der Beteiligten wirksam werden. Die Politik sozialer Probleme erscheint in Formen sozialer Kontrolle (Groenemeyer 2001b, S. 148f.), weil eine Allgemeinheit hieran beteiligt ist. Soziale Kontrolle wird als Effekt erfolgreicher Problematisierung gesehen (Groenemeyer 2001a), insofern Instanzen sozialer Kontrolle die Problematisierten bearbeiten und eine Problemlage auf die Kapazitäten ihres Ressorts übersetzen - Polizei, Gericht, Strafvollzug. Dabei definieren sie Problemgruppen (z.B. Naplava 2008). Gleichzeitig zeugt das Ringen 
der Zugehörigen zum problematisierten Kollektiv, Problemzuschreibungen von sich fernzuhalten, gerade von der Dynamik, mit der Allgemeinheit und Problemgruppe einander festzuzurren versuchen. So ist das problematisierte Kollektiv eine Kategorie im Übergang zwischen beiden, wie etwa Ammann Dula (2019) am Beispiel albanischer Einwander*innen in der Schweiz zeigt, die unermüdlich Abgrenzungen zur Problemgruppe vornehmen und ihre Position als Allgemeinheit unterstreichen, indem sie ihr Engagement der Entproblematisierung widmen. Hieran sollten sich auch wissenschaftliche Zugänge beteiligen.

Danksagung Ich danke den Kolleg*innen aus dem Kreis der Redaktion der Sozialen Probleme für die wertvollen Kommentare und Anregungen.

Funding Open Access funding enabled and organized by Projekt DEAL.

Open Access Dieser Artikel wird unter der Creative Commons Namensnennung 4.0 International Lizenz veröffentlicht, welche die Nutzung, Vervielfältigung, Bearbeitung, Verbreitung und Wiedergabe in jeglichem Medium und Format erlaubt, sofern Sie den/die ursprünglichen Autor(en) und die Quelle ordnungsgemäß nennen, einen Link zur Creative Commons Lizenz beifügen und angeben, ob Änderungen vorgenommen wurden.

Die in diesem Artikel enthaltenen Bilder und sonstiges Drittmaterial unterliegen ebenfalls der genannten Creative Commons Lizenz, sofern sich aus der Abbildungslegende nichts anderes ergibt. Sofern das betreffende Material nicht unter der genannten Creative Commons Lizenz steht und die betreffende Handlung nicht nach gesetzlichen Vorschriften erlaubt ist, ist für die oben aufgeführten Weiterverwendungen des Materials die Einwilligung des jeweiligen Rechteinhabers einzuholen.

Weitere Details zur Lizenz entnehmen Sie bitte der Lizenzinformation auf http://creativecommons.org/ licenses/by/4.0/deed.de.

\section{Literatur}

Albrecht, Günther. 2001. Gesellschaftliche Konstruktion von Realität und Realität von Konstruktionen. Soziale Probleme 12:116-145.

Ammann Dula, Evelyn. 2019. Handlungsstrategien im Kontext intersektionaler Ungleichheiten. Eine biograpieanalytische Untersuchung zweier Geschwister einer albanischen Familie in der Schweiz. In Die Problematisierung sozialer Gruppen in Staat und Gesellschaft, Hrsg. Dörte Negnal, 239-256. Wiesbaden: Springer VS.

Becker, Howard S. 2014. Außenseiter. Zur Soziologie abweichenden Verhaltens, 2. Aufl., Wiesbaden: Springer VS. engl. Orig.: Outsiders. Studies in the Sociology of Deviance. New York: Free Press.

Bellebaum, Alfred, und Hans Braun (Hrsg.). 1974. Empirische Befunde. Reader Soziale Probleme, Bd. I. Frankfurt am Main: Herder \& Herder.

Blumer, Herbert. 1971. Social problems as collective behavior. Social Problems 18:298-306.

Cohen, Stanley. 1972. Folks devils and moral panics. London: McGibbon \& Kee.

Dollinger, Bernd. 2010. Doing Social Problems in der Wissenschaft. Sozialpädagogik als disziplinäre Form der Problemarbeit. In Doing Social Problems. Mikroanalysen der Konstruktion sozialer Probleme und sozialer Kontrolle in institutionellen Kontexten, Hrsg. Axel Groenemeyer, 105-123. Wiesbaden: Springer VS.

Fürstenberg, Friedrich. 1965. Randgruppen in der modernen Gesellschaft. Soziale Welt 16:236-245.

Goffman, Erving. 1973a. Asyle. Über die soziale Situation psychiatrischer Patienten und anderer Insassen. Frankfurt am Main: Suhrkamp.

Goffman, Erving. 1973b. Wir alle spielen Theater. Die Selbstdarstellung im Alltag, 2. Aufl., München: Piper.

Goffman, Erving. 1990. Stigma. Notes on the management of spoiled identity. London: Penguin Books. 
Gottschalk, Ines, und Dilek Aysel Tepeli. 2019. Etablierten-Außenseiter-Beziehungen im Ankunftsstadtteil Dortmunder Nordstadt. In Die Problematisierung sozialer Gruppen in Staat und Gesellschaft, Hrsg. Dörte Negnal, 63-81. Wiesbaden: Springer VS.

Groenemeyer, Axel. 2001a. Soziologische Konstruktionen sozialer Probleme und gesellschaftliche Herausforderungen - Eine Einführung. Soziale Probleme 12:5-27.

Groenemeyer, Axel. 2001b. Von der Sünde zum Risiko? - Bilder abweichenden Verhaltens und die Politik sozialer Probleme am Ende des Rehabilitationsideals. Soziale Probleme 12:146-182.

Groenemeyer, Axel. 2010. Doing Social Problems - Doing Social Control. Mikroanalysen der Konstruktion sozialer Probleme und sozialer Kontrolle in institutionellen Kontexten. Ein Forschungsprogramm. In Doing Social Problems. Mikroanalysen der Konstruktion sozialer Probleme und sozialer Kontrolle in institutionellen Kontexten, Hrsg. Axel Groenemeyer, 13-56. Wiesbaden: Springer VS.

Groenemeyer, Axel. 2012. Soziologie sozialer Probleme. Fragestellungen, Konzepte und theoretische Perspektiven. In Handbuch soziale Probleme, 2. Aufl., Bd. 1, Hrsg. Günther Albrecht, Axel Groenemeyer, 17-116. Wiesbaden: Springer VS.

Groenemeyer, Axel, und Silvia Wieseler (Hrsg.). 2008. Soziologie sozialer Probleme und sozialer Kontrolle. Realitäten, Repräsentationen und Politik. Wiesbaden: Springer VS.

Gruhlich, Julia. 2019. Schwarze Männlichkeiten. Zur Problematisierung der Problematisierung. In Die Problematisierung sozialer Gruppen in Staat und Gesellschaft, Hrsg. Dörte Negnal, 43-62. Wiesbaden: Springer VS.

Gusfield, Joseph R. 1986. Symbolic crusade. Status politics and the American temperance movement, 2. Aufl., Urbana: University of Illinois Press.

Gusfield, Joseph R. 1989. Constructing the ownership of social problems: fun and profit in the welfare state. Social Problems 36:431-441.

Hess, Henner. 1993. Kriminologen als Moralunternehmer. In Vom Guten, das noch stets das Böse schafft, Hrsg. Lorenz Böllinger, Rüdiger Lautmann, 329-347. Frankfurt am Main: Suhrkamp.

Hess, Henner. 2015. Probleme der sozialen Kontrolle. In Die Erfindung des Verbrechens, Hrsg. Henner Hess, 19-40. Wiesbaden: Springer VS.

Hirschauer, Stefan. 2017. Un/doing Differences: Praktiken der Humandifferenzierung. Weilerswist: Velbrück.

Holstein, James, und Gale Miller. 1993. Reconstituting the Constructionist Program. In Reconsidering Social Constructionism Debates in Social Problems Theory, 241-250. Hawthorne: Aldine.

Karstedt, Susanne. 1975. Soziale Randgruppen und soziologische Theorie. In Stigmatisierung - zur Produktion gesellschaftlicher Randgruppen, Bd. 1, Hrsg. Manfred Brusten, Jürgen Hohmeier, 169-193. Neuwied, Darmstadt: Luchterhand.

Keysers, Verena, Joanna Meißner, Jo Reichertz, und Nils Spiekermann. 2019. Die Einlassanlage von Fußballstadien als Stahl gewordener Imperativ. Situative und übersituative Praktiken des Problematisierens beim Fußball. In Die Problematisierung sozialer Gruppen in Staat und Gesellschaft, Hrsg. Dörte Negnal, 167-191. Wiesbaden: Springer VS.

Kitsuse, John I., und Malcom Spector. 1973. Toward a sociology of social problems: social conditions, value-judgements, and social problems. Social Problems 20:407-419.

Lautmann, Rüdiger. 2019. Paradoxe Effekte einer Problemgruppenkonstruktion: Respression und Emanzipation der Homosexuellen seit 1850. In Die Problematisierung sozialer Gruppen in Staat und Gesellschaft, Hrsg. Dörte Negnal, 295-319. Wiesbaden: Springer VS.

Miller, Gale, und James A. Holstein. 1989. On the sociology of social problems. In Perspectives on social problems, 1-16. Greenwich: JAI Press.

Naplava, Thomas. 2008. Jugendliche Intensivtäter als Kriminalitätsproblem und Problemkonstruktion. In Soziologie sozialer Probleme und sozialer Kontrolle. Realitäten, Repräsentationen und Politik, Hrsg. Axel Groenemeyer, Silvia Wieseler, 193-214. Wiesbaden: VS.

Negnal, Dörte. 2016. Die Konstruktion einer Problemgruppe. Eine Ethnografie über russischsprachige Inhaftierte im Jugendstrafvollzug. Weinheim: Beltz Juventa.

Negnal, Dörte. 2019a. Geschlossene Gesellschaft. Über die Problematisierung von Gruppen in Haft. In Geschlossene Institutionen - geschlossene Gemeinschaften, Hrsg. Anke Neuber, Franz Zahradnik, 168-181. Weinheim, Basel: Beltz Juventa.

Negnal, Dörte. 2019b. Die Problematisierung sozialer Gruppen. In Die Problematisierung sozialer Gruppen in Staat und Gesellschaft, Hrsg. Dörte Negnal, 15-42. Wiesbaden: Springer VS.

Peters, Helge. 2000. Soziale Kontrolle. Zum Problem der Normkonformität in der Gesellschaft. Wiesbaden: Springer VS. 
Peters, Manuel. 2009. Zur sozialen Praxis der (Nicht-)Zugehörigkeiten. Die Bedeutung zentraler Theorien von Bourdieu und Goffman für einen Blick auf Migration, Zugehörigkeit und Interkulturelle Pädagogik. Oldenburg: BIS.

Quensel, Stephan. 1970. Wie wird man kriminell? Verlaufsmodell einer fehlgeschlagenen Interaktion zwischen Delinquenten und Sanktionsinstanz. Kritische Justiz 3:375-382.

Rheinberger, Hans-Jörg. 1997. Toward a history of Epistemic things: synthesizing proteins in the test tube. Stanford: University Press.

Rheinberger, Hans-Jörg. 2006. Epistemologie des Konkreten. Studien zur Geschichte der modernen Biologie. Frankfurt am Main: Suhrkamp.

Sacks, Harvey. 1972. On the analyzability of stories by children. In Directions in sociolinguistics - the ethnography of communication, Hrsg. John J. Gumperz, David Hymes, 325-345. New York: Holt, Rinehart and Winston.

Sacks, Harvey. 1992. Lecture 6 - the MIR membership categorization device. In Lectures on conversation, Bd. 1, Hrsg. Gail Jefferson, 40-48. Oxford: Blackwell.

Scheerer, Sebastian. 1978. Der politisch-publizistische Verstärkerkreislauf. Kriminologisches Journal 10(3):223-227.

Scheerer, Sebastian. 2000. „Soziale Kontrolle“ - Schöner Begriff für böse Dinge? In Soziale Kontrolle, Hrsg. Helge Peters, 153-169. Wiesbaden: Springer VS.

Scheffer, Thomas. 2008. Zug um Zug und Schritt für Schritt. Annäherungen an eine transsequentielle Analytik. In Theoretische Empirie. Zur Relevanz qualitativer Forschung, Hrsg. Herbert Kalthoff, Stefan Hirschauer, und Gesa Lindemann, 368-398. Frankfurt am Main: Suhrkamp.

Scheffer, Thomas, Christiane Howe, Eva Kiefer, Dörte Negnal, und Yannik Porsché. 2017. Polizeilicher Kommunitarismus. Zur Praxis urbaner Kriminalprävention. Frankfurt am Main, New York: Campus.

Scherr, Albert, und Lena Sachs. 2019. Sinti und Roma als Problemgruppe? Problematisierung und Entproblematisierung im Kontext von Nicht-Wissen und politischer Correctness. In Die Problematisierung sozialer Gruppen in Staat und Gesellschaft, Hrsg. Dörte Negnal, 277-293. Wiesbaden: Springer VS.

Schetsche, Michael. 1996. Die Karriere sozialer Probleme. Berlin: DeGruyter.

Schetsche, Michael. 2001. Meta-Theorie und Analyseprogramm - Zum Doppelstatus der relativistischen Problemtheorie. Soziale Probleme 12:28-44.

Schetsche, Michael. 2014. Empirische Analyse sozialer Probleme. Das wissenssoziologische Programm, 2. Aufl., Wiesbaden: Springer VS.

Stallberg, Friedrich. 2008. Russlanddeutsche als Kleinstadtproblem - Thematisierungsformen im Spannungsfeld von Integrationserwartungen und religiös bedingter Isolation. In Soziologie sozialer Probleme und sozialer Kontrolle. Realitäten, Repräsentationen und Politik, Hrsg. Axel Groenemeyer, Silvia Wieseler, 552-585. Wiesbaden: VS.

Thalheim, Vinzenz. 2019. Ultras - was hat das noch mit Fußball zu tun? Zeitschrift für Jugendkriminalrecht und Jugendhilfe 2:113-120.

Wehrheim, Jan. 2000. Ein fast ignoriertes Überwachungsdrama. Zur technischen, politischen und gesellschaftlichen Realität von Closed Circuit Television. In Forum Wissenschaft, 34-40.

Wehrheim, Jan. 2018. Kritik der Versicherheitlichung. Thesen zur (sozialwissenschaftlichen) Sicherheitsforschung. Kriminologisches Journal 50:211-221.

Weis, Kurt. 1999. Gewalt und Sport: Rechte Rowdies in rechter Gesellschaft? In Handbuch soziale Probleme, Hrsg. Günther Albrecht, Axel Groenemeyer, und Friedrich W. Stallberg, 556-574. Opladen, Wiesbaden: Westdeutscher Verlag. 